\title{
MULTI-LEAD ECG COMPRESSION BASED ON COMPRESSIVE SENSING
}

\author{
Javad Afshar Jahanshahi
}

\begin{abstract}
Compressed Sensing (CS) has been considered a very effective means of reducing energy consumption at the energy-constrained wireless body sensor networks for monitoring the multi-lead Electrocardiogram (MECG) signals. This paper develops the compressed sensing theory for sparse modeling and effective multi-channel ECG compression. A basis matrix with Gaussian kernels is proposed to obtain the sparse representation of each channel, which showed the closest similarity to the ECG signals. Thereafter, the greedy orthogonal matching pursuit (OMP) method is used to obtain the sparse representation of the signals. After obtaining the sparse representation of each ECG signal, the compressed sensing theory could be used to compress the signals as much as possible. Following the compression, the compressed signal is reconstructed utilizing the greedy orthogonal matching pursuit (OMP) optimization technique to demonstrate the accuracy and reliability of the algorithm. Moreover, as the wavelet basis matrix is another sparsifying basis to sparse representations of ECG signals, the compressed sensing is applied to the ECG signals using the wavelet basis matrix. The simulation results indicated that the proposed algorithm with Gaussian basis matrix reduces the reconstruction error and increases the compression ratio.
\end{abstract}

Keywords - $\quad$ Compressed sensing, Electrocardiogram signal, Gaussian matrix, Sparse representation.

\section{INTRODUCTION}

$\mathbf{T}$ he ambulatory monitoring of bioelectric signals has received considerable attention from different scholars in recent years. Such monitoring procedures offer several advantages, such as constant patient observation, increased patient mobility, and decreased healthcare costs [1]. However, there are difficulties with these devices, such as wearable device size, energy consumption, and energy costs. The energy consumption of these devices has long been considered the most important challenge. Moreover, in ambulatory environments, there is a strong need for the proper management of a large volume of bioelectric signals, generated by the health monitoring devices/sensors [1]. Hence, the lossy compression methods have captured

Universidad Católica Los Ángeles de Chimbote, Instituto de Investigación, Chimbote, Perú. considerable attention. These devices are mainly used to reduce the transmitted information while ensuring maximum signal quality. In this regard, compressive sensing (CS) is one of the most popular methods of compression, which is built-in and well suited to the energy-efficient wearable and mobile devices/sensors; this method is used to reduce the data to be stored and the data required by the micro-controllers and the analog-digital converters [2]. Therefore, [3] suggested that it is possible to utilize compressive sensing to reduce energy consumption. It helped to decrease the hardware complexity of the wireless body sensor network-enabled ECG monitors as well [4]. Today, the graphical representation of the electrical activities of the heart or ECG (electrocardiography) signals plays a substantial role in determining, diagnosing, and even preventing various cardiac diseases. These signals have to be recorded in the long-run (continuously for one or two weeks or even longer) for the accurate diagnosis of various diseases. The storage, transmission, or processing of this large volume of information are extremely difficult and require very large storage space and considerable time, especially when multiple ECG electrodes sense a high-resolution signal. Hence, the bioelectric signals have to be compressed using the compression methods [1, 3-4].

In recent years, many methods have been used for the optimal compression of electrocardiogram signals at a high compression ratio. Before the introduction of the compressed sensing theory, the methods were mainly based on direct compression methods. Methods such as AZTEC (amplitude zone epoch coding), CORTES (coordinate reduction time encoding), and SAPA (scan along with polygonal approximation) [5-7] are among the direct compression methods, which function based on the direct extraction of significant signal samples and omission of the insignificant parts. In these methods, most parts of the signal are omitted. Besides, sometimes even the significant parts are considered eliminated and are omitted along with the other parts of the data. Other compression methods involve the use of transforms to represent the signal to another sparse space. Transforms such as the Fourier transform, discrete cosine transform, and discrete wavelet transform are among these methods [8-11]. However, the degree of compression of these transforms is not satisfactory and none of these transforms forms an acceptable basis matrix for the electrocardiogram signals due to their lack of similarity to them. The third electrocardiogram signal compression method involves the use of the parameter extraction 
methods, which are a combination of the direct compression method and the mentioned transforms such as the peak picking (PP) and vector quantization (VQ) methods [12-13].

Compressed sensing is a new method for compressing different types of signals, and it has been highly popular and commonly used in recent years [3-5]. In this method, sampling and compression of sparse signals are simultaneously carried out. Signals with sparse representations or almost sparse representations are reconstructed with very fewer samples than the Nyquist sampling method. In the past several years, compressed sensing has been used for the compression of electrocardiogram signals using a single electrode or multiple electrodes (i.e. single or multi-lead ECG). In [14], Compressed sensing is used to compress the ECG signals. Compressed sensing method has also been used to compress 12 to 15 multi-lead electrocardiogram signals in recent years [15-18]. In all of these references, the wavelet basis matrix is used for the sparse representation of ECG signals. As stated earlier, the wavelet basis matrix is not highly similar to the electrocardiogram signals and it is not an ideal option for sparsifying electrocardiogram signals. In [19], it is demonstrated that the ECG signals have the closest similarity to the Gaussian signals, and then these signals are optimally modelled using the Gaussian functions. Given this similarity, a basis matrix using Gaussian kernels is formed for sparsifying the ECG signal. Finally, the optimization problem has to be solved to obtain the sparse representation of the ECG signal.

Inspired by the promising results of the aforementioned methods, inthis paper, a new method is proposed for modelling the ECG signals and compressing these signals based on the compressed sensing theory. First, the sparse representation of the signals is required to implement the method. The sparse representation of each signal is obtained using a basis matrix with Gaussian kernels. Thereafter, the compressed sensing is used for signal compression with very fewer samples than the original signal.

This paper is organized as follows; in section 2, the latest related works are reviewed in brief. In section 3, the compressed sensing theory is introduced briefly. In section 4 , the compressed sensing theory is applied to the real and artificial ECG signals. Afterward, in section 5 , the results of the simulation and efficiency of the proposed theory are presented, and the summary and conclusions are provided in section 6 .

\section{RELATED WORKS}

In wireless body sensor networks, sensors have limited computation capability and power resources without the assistance of any established infrastructures; many

Copyright (C) Authors studies in the literature validate the potential of CS for energy-efficient ECG compression [20-24]. In this sense, the proposed algorithm in [25] assigns not only more weights to important parts of the ECG signals but also low priorities to other parts. Subsequently, by integrating an iterative weighted CS-based method, the proposed algorithm effectively solves the 1_1 minimization problem. This approach ignores the dependencies of the signal of interest in each window to the signal of the previous epoch (temporal correlations). For the recovery of the ECG signals, a modified iterative algorithm has been proposed in [26], which assumes that specific prior information about the signals is typically available. Hence, the performance of the recovery algorithm is directly influenced by the accuracy of the prior information of the signal and the particular application, which leads to somewhat accurate reconstruction and compression rates. The authors of [27] proposed a dictionary learning-based CS approach for sparse recovery of the ECG signals instead of the wavelet or DCT dictionaries. According to the morphology of the ECG signals, three different dictionaries are trained and applied for the recovery of the corresponding signal type. It has been confirmed that dictionary learning approaches require a huge amount of signal transmission energy and impose a high computational load on the resource constraints nodes [28-29]. Another work that considers temporal correlation among the ECG signals is [30], in which a principle component analysis (PCA) based algorithm has been developed to choose the necessary features of a signal (i.e., eigenvalue or principle components), which facilitate signal reconstruction without a loss of signal morphologies. In this work, a complicated multistage pre-processing step should be considered, which renders the algorithm inappropriate for real-time applications. Recently, a BSBL framework based CS has been proposed to efficiently exploit the spatial correlation and to accurately recover non-sparse physiological signals [31]. Since the BSBL framework is too slow in time for wearable wireless ECG monitoring systems, [32] have utilized the ADMM solution to improve the efficiency of their method.

In this paper, compressed sensing is used for multi-lead ECG signal compressions. First, in order to obtain a sparse representation of the ECG signals, a dictionary of Gaussian kernels is created to meet the right condition of utilizing compressed sensing theory. It will show that using the Gaussian basis improves the compression ratio. Thereafter, based on compressed sensing, a sensing matrix is created to meet the theory conditions and helps to more compress the signals with very fewer samples. Finally, the signals are reconstructed using the orthogonal matching pursuit optimization method. Simulation results verify that the proposed algorithm achieves greater reconstruction accuracy with a smaller number of required transmissions, lower computational 
complexity, and smaller reconstruction errors, as compared to the CS-based recovery methods.

\section{A BRIEF REVIEW OF THE COMPRESSIVE SENSING}

Compressive Sensing (CS) provides a simultaneous sensing and compression framework [2], enabling a potentially significant reduction in the sampling and computation costs at a sensor with limited capabilities. CS theory shows that a signal with a sparse transform representation can be reconstructed from a small set of incoherent random projections. Three mathematicians established the mathematical fundamentals and bases of the compressed sensing method in 2006 [2]. They suggested that perfect reconstruction may still be possible when the sample-rate criterion is not satisfied, provided that some conditions hold for the underlying signals. They proved that if a signal is exactly or approximately sparse in some appropriate domain, it can be reconstructed with even fewer samples than what the sampling theorem requires. Sparsity means that most of the elements of the signal are zero or nearly zero, leading to a reduced degree of freedom in signal representation. This idea is the basis of CS theory.

The condition of sparsity of the signals in the compressed sensing theory is as important as the bandwidth condition in the Nyquist theory [33-34]. In fact, if this condition is not met, the compressed sensing theory cannot be used. From the mathematical point of view, signal $x \in R^{\wedge}(N \times 1)$ is called $k$-sparse if only $k$ signal samples are nonzero and the rest are zero. Most of the existing signals, including electrocardiogram signals, lack the sparsity features. Therefore, a basis matrix like $\Phi \in \mathrm{R}^{\wedge}(\mathrm{N} \times \mathrm{M})$ has to be defined to obtain the sparse representation of the signal. In this case, a signal like $\mathrm{x}$ is called $\mathrm{k}$-sparse because it can be written as $\mathrm{x}=\Phi \mathrm{c} \quad$ where $\quad \mathrm{c} \quad \epsilon \rrbracket \quad \mathrm{R} \rrbracket^{\wedge}(\mathrm{M} \times 1)$ and $\|\mathrm{c}\| \_\leq \mathrm{k}$. Electrocardiogram signals are sampled simultaneously with multiple electrodes to obtain multi-lead ECG signals (i.e. 12 to 15 electrodes). Therefore, the sparse representation of the multi-lead ECG signals is written as follows;

$$
\mathrm{X}=\Phi \mathrm{C}
$$

Where $\mathrm{X}=\left(\mathrm{x}_{1}, \mathrm{x}_{2}, \ldots, \mathrm{X}_{\mathrm{S}}\right) \in \mathrm{R}^{\mathrm{N} \times \mathrm{S}}$ is a matrix containing $\mathrm{S}$ ECG signals and each column of matrix $X$ shows one lead (channel). Besides, $\Phi=\left(\phi_{1}, \phi_{2}, \ldots, \phi_{\mathrm{M}}\right) \in \mathrm{R}^{\mathrm{N} \times \mathrm{M}}$ is the basis matrix and $\mathrm{C}=\left(\mathrm{c}_{1}, \mathrm{c}_{2}, \ldots, \mathrm{c}_{\mathrm{s}}\right) \in \mathrm{R}^{\mathrm{M} \times \mathrm{S}}$ is the sparse representation of each ECG signal, where $c_{j}$ is the sparse representation of lead $x_{j}$. By acquisition of the sparse representation of the signal, the primary condition of the compressed sensing is met and the signals are compressed as follows;

$$
\mathrm{Y}=\mathrm{AX}
$$

Where $\mathrm{A} \in \mathcal{R}^{\mathrm{mxN}}$ is the measurement matrix and $\mathrm{Y}=\left(\mathrm{y}_{1}, \mathrm{y}_{2}, \ldots, \mathrm{y}_{\mathrm{s}}\right) \in \mathcal{R}^{\mathrm{mxS}}$ is the compressed signal for each ECG lead. The other condition for using compressed sensing is preparing the proper measurement matrix. In [35], the authors proved that if the entries in the measurement matrix are independent and uniformly distributed samples of a Gaussian distribution (i.e., i. i.d), the measurement matrix satisfies the most important condition in the compressed sensing, i.e. the RIP (Restricted Isometry Property) condition. According to this condition, if $\delta \in(0,1)$, a matrix like $\mathrm{A} \in \mathcal{R}^{\mathrm{m \times N}}$ meets the RIP condition with a degree of $\mathrm{k}$ as follows

$$
\left(1-\delta_{\mathrm{k}}\right)\|\mathrm{x}\|_{2}^{2} \leq\|\mathrm{Ax}\|_{2}^{2} \leq\left(1+\delta_{\mathrm{k}}\right)\|\mathrm{x}\|_{2}^{2}
$$

The mathematical results indicate that compressed sensing can be used if the measurement matrix satisfies these conditions. As a result, an $\mathrm{N}$-dimensional signal is transformed into an m-dimensional compressed signal and $(\mathrm{m} \ll \mathrm{N})$. The proper value of $\mathrm{m}$ has to be selected such that the equation 4 is satisfied.

$$
\mathrm{m} \geq \mathrm{u} \times \mathrm{k} \times \log (\mathrm{N} / \mathrm{k})
$$

where $=\frac{1}{2} \log (\sqrt{24}+1) \approx 0.28$. There are several optimization methods for signal reconstruction. Each of these methods can be selected to solve a problem depending on their applications. As stated before, for solving problems like there are some Greedy methods such as Matching Pursuit (MP), Orthogonal Matching Pursuit (OMP) [36], and Compressive Sampling Matching Pursuit (CoSaMP) [37].

\section{The PROPOSED CS BASED ALGORITHM}

In this section, an efficient algorithm is proposed for the compression of multi-lead ECG signals. As stated, ECG signals typically lack the sparsity features and a proper basis matrix is needed for the sparse representation of these signals. First, a basis matrix has to be built-in with the Gaussian kernels to obtain the sparse representation of the signal. Thereafter, the $\mathrm{N}$ dimensional original signal is mapped to $\mathrm{m}$ dimensions $(\mathrm{m} \ll \mathrm{N})$ using a proper measurement matrix that meets the RIP condition. It is not easy to demonstrate that the measurement matrix meets the important RIP condition as it needs to prove complicated and difficult relations. Hence in the literature, it is usually tried to replace the RIP condition with its other soft equivalents to prove the functionality of the measurement matrix. To this end, the coherency concept is used. In other words, the similarity between the columns of the measurement matrix is calculated via equation (5). 


$$
\mu(A)=\max _{1 \leq i \leq j \leq n} \frac{\left|\left\langle\alpha_{i}, \alpha_{j}\right\rangle\right|}{\left\|\alpha_{i}\right\|_{2}\left\|\alpha_{j}\right\|_{2}}
$$

where $\alpha_{i}$ and $\alpha_{j}$ denote the columns of the measurement matrix. If the correlation is adequately low, the reconstruction of the compressed signal is guaranteed with minimum reconstruction error [35]. Besides, the correlation is always in the range of $\left[\sqrt{\frac{\mathrm{N}-\mathrm{m}}{\mathrm{m}(\mathrm{N}-1)}}, 1\right]$. The sensing matrix $\Psi=A \Phi$ has to meet the RIP conditions as well.

The first step to compress the selected signals is acquiring the sparse representation of the signals. To this end, a basis matrix with Gaussian kernels has to be created. Therefore, an adequate number of Gaussian functions with various means and variances are required. A Gaussian kernel is expressed as follows.

$$
g\left(\frac{s-a}{b}\right)=e^{\frac{(a-a)^{2}}{b^{2}}}
$$

Where $\mathbf{a}$ and $\mathbf{b}$ are the shift and scaling parameters, respectively. In order to create a basis matrix that suits the sparse representation of the ECG signals, the proper shift and scale ranges have to be selected. One of the characteristics of the ECG signals is that the R peak in these signals is fully prominent and identifiable. This feature is used to find the proper range of the shift values. For instance, the number of samples in these ranges is calculated by finding the peak $R$ in the signal and knowing that the respective range lengths of the $\mathrm{P}-\mathrm{R}$ and $\mathrm{R}-\mathrm{T}$ peaks at the $360 \mathrm{~Hz}$ sampling frequency in a normal ECG signal are at most 200 and $450 \mathrm{~ms}$. The number of samples in ranges $\mathrm{P}-\mathrm{R}$ and $\mathrm{R}-\mathrm{T}$ is 72 and 162, respectively. Therefore, the shift associated with the Gaussian kernels has to fall in the range of $\left[S_{R}-72, S_{R}+162\right]$ to fully cover one period of the ECG signal. In this range, $S_{R}$ is the index for peak R. According to [19], the suitable range for the scale of the ECG signals at the sampling frequency $360 \mathrm{~Hz}$ is $[0.06-0.02]$. The size of the columns of the basis matrix $\Phi$ equals to $\mathrm{M}=\mathrm{r}^{\mathrm{X}} \mathrm{t}$, where $\mathrm{r}$ and $\mathrm{t}$ show the number of different shift values and scales of the Gaussian kernels, respectively. Finally, the basis matrix is obtained as follows:

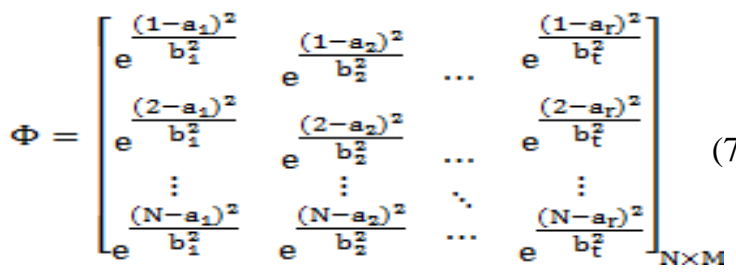

The sparse representation of the signal is obtained after creating the basis matrix. Various methods such as the convex and greedy optimization methods are available for obtaining the sparse representations [36-37]. In this paper, the greedy OMP optimization method is used [36].

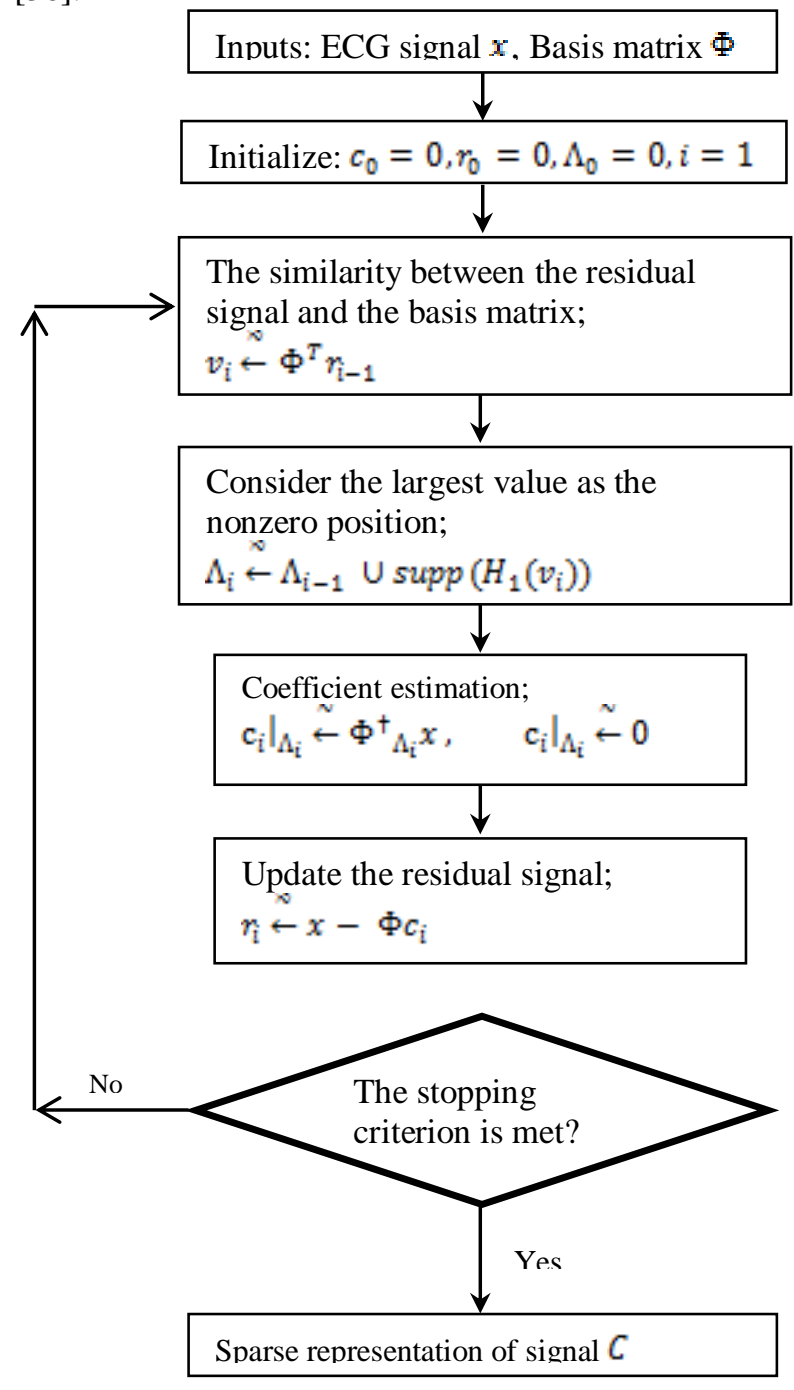

Figure (1): The block diagram of the proposed method based on the OMP algorithm

OMP is one of the best and most commonly used methods for solving optimization problems in the compressed sensing theory and reconstruction of the compressed signals.

The OMP algorithm starts with finding a column in the basis matrix $\Phi$, which shows the closest similarity with the selected signal. In this regard, the algorithm finds the similarity between the basis matrix and the signal residue. The signal residue is obtained by deducting the component calculated in the previous stage from the original signal. The different steps of implementing the OMP algorithm are depicted in a block diagram 
depicted in Figure (1). In this block diagram, $\mathrm{H}_{\mathrm{k}}(\mathrm{x})$ represents the hard thresholding operator on $\mathrm{X}$, which sets all the input components (except for the $\mathrm{k}$ inputs from $\mathrm{x}$ ), which have the longest range, to zero [35].

The OMP algorithm is used to obtain the sparse representation of all leads the ECG signal separately. After obtaining the sparse representation of each single lead and putting each one in the columns of a matrix, it is possible to apply the compressed sensing. Next, the measurement matrix that satisfies the RIP condition is created. After building this matrix, compression is carried out using equation (8).

$$
\mathrm{Y}=\mathrm{A} \Phi \mathrm{C}
$$

In the next section, the simulation results experiments verify that low exists between the columns of the sampling matrix $\Phi$ and thus it satisfies the RIP condition.

\section{DISCUSSION AND SIMULATION RESULTS}

In this section, the results of the proposed algorithm simulations are presented for the compression of electrocardiogram signals. This technique is used to compress the artificial and real signals. Afterward, the mean reconstruction error is calculated for the original artificial signal and the reconstructed signal. The diagram of the compression ratio versus the mean squared error is also presented. In this diagram, the dimensions of the original signal and the reconstructed signal are linked to the reconstruction error. Finally, the method proposed in [11], in which the wavelet basis matrix is used, is compared with the proposed algorithm. In order to find out which algorithm is more efficient in terms of computational complexity, reconstruction error comparison is carried out between the algorithms. The configuration of the used system is Intel Core2Duo, 2.53GHz, P8700, 4GB RAM in 64BitMatlab R20013b platform. The PTB database includes 15-channel signals collected from 290 patients at the sampling frequency $\mathrm{f}_{\mathrm{s}}=1 \mathrm{kHz}$ and resolution rate of 16 bits. In the simulation results, all of the multi-channel signals in these two databases were used. Since WBAN networks comply mostly with the frequencies lower than $369 \mathrm{~Hz}$, every ECG signal was re-sampled at the frequency $250 \mathrm{~Hz}$. This is due to the fact that the ECG signals in the wireless body sensor networks are normally sampled at the frequency $250 \mathrm{~Hz}$ [38-41]. The simulation results show the superiority of the Gaussian basis matrix and the proposed algorithm to the wavelet basis matrix.

\subsection{Compression of the Artificial ECG Signal}

In this subsection, due to the availability of real multilead ECG signals, we are going to evaluate the

Copyright (C) Authors performance of the proposed algorithm on artificial ECG signals. The advantage of the availability of the original signal is the possibility of the addition of noises and the analysis of the algorithm performance in different conditions. The actual error is also obtained through this method. So, in [33] the process of creating artificial electrocardiogram signals is described.

Firstly, a period of the artificial single-lead ECG signal is created using a model of artificial cardiac single production in [33]. Thereafter, the standard Kalman filter, which is introduced in [40], provided the artificial noise using the time-varying AR variables. The produced noise from this method has all the characteristics of the noise of the real ECG signal. The simplified discrete model for the generation of artificial ECG signals is as follows.

$$
\left\{\begin{array}{c}
\theta_{\mathrm{k}+1}=\left(\theta_{\mathrm{k}}+\delta \omega\right) \bmod (2 \pi) \\
\mathrm{z}_{\mathrm{k}+1}=-\sum_{\mathrm{i} \in[\mathrm{P}, \mathrm{Q}, \mathrm{R}, \mathrm{S}, \mathrm{T}\}} \delta \frac{\mathrm{a}_{\mathrm{i}} \omega}{\mathrm{b}_{\mathrm{i}}^{2}} \Delta \theta_{\mathrm{i}} \exp \left(-\frac{\Delta \theta_{\mathrm{i}}^{2}}{2 \mathrm{~b}_{\mathrm{i}}^{2}}\right)+\mathrm{z}_{\mathrm{k}}+\eta
\end{array}\right.
$$

Where $\Delta \theta_{-} \mathrm{i}=\left(\theta_{-} \mathrm{k}-\theta_{-} \mathrm{i}\right) \bmod (2 \pi)$ and $\eta$ is the additive noise to the signal and the sum on $\mathrm{i}$ consists of the five dominant waves in the ECG signal $(\mathrm{P}, \mathrm{Q}, \mathrm{R}, \mathrm{S}, \mathrm{T})$. The parameters required for equation (9) are listed in Table (1).

Table (1): The parameters for producing the artificial

\begin{tabular}{|c|c|c|c|c|c|}
\hline $\begin{array}{l}\text { Index } \\
\text { (i) }\end{array}$ & $\mathrm{P}$ & $Q$ & $\mathrm{R}$ & S & $\mathrm{T}$ \\
\hline$\theta_{\mathrm{i}}($ rads. $)$ & $-\pi / 3$ & $-\pi / 12$ & 0 & $\pi / 12$ & $\pi / 2$ \\
\hline$a_{i}$ & 1.2 & -5 & 30 & 7.5 & 0.7 \\
\hline $\mathrm{b}_{\mathrm{i}}$ & 0.25 & 0.1 & 0.1 & 0.1 & 0.4 \\
\hline
\end{tabular}
electrocardiogram signal

The artificial ECG signal is generated at the desired sampling frequency using the dynamic equations and the parameters. For instance, a period of this signal is depicted in Figure (2) at the $500 \mathrm{~Hz}$ sampling frequency. The sparse representation of the signal, which was obtained using a basis matrix $\Phi$ with a size of $3000 \times 6000$, is presented in Figure (3). The nonzero coefficients are obtained using the OMP algorithm, in which the signal sparsity is 55 . The measurement matrix A has a size of $130 \times 3000$. The inputs to this matrix are obtained from the Gaussian i.i.d distributions with a mean of zero and a variance of 1. Based on equation (4), number 130 is selected, which equals the size of the compressed signal. The sparse representation and the reconstructed one are presented in Figure (3). Hence, the OMP algorithm is used to reconstruct the sparse representation of the ECG signal. As seen in Figure (3), 
the reconstructed sparse representation is aligned with the original sparse representation at most points, and it clearly shows adequate reconstruction of the signal. After reconstructing the sparse coefficients, the ECG signal is recovered using these coefficients. As seen in Figure (2), the reconstructed ECG signal is fully aligned with the original signal, reflecting its accurate reconstruction. In Figure (4), the original signal is depicted with its sparse representation. According to Figure (4), the sparse coefficients are fully aligned with the original signal, while the OMP algorithm properly determines the positions of the important coefficients and accurately estimates their sizes. Figure (5) also presents the compressed ECG signal samples.

Another signal is shown in Figure (6) with an increase in noise to demonstrate the effectiveness of the proposed algorithm for further smoothing the signal and reducing the noise. The signal to noise ratio in Figure (6) decreased by $7 \mathrm{db}$ as compared to Figure (2). As seen in this figure, the reconstructed signal is more smoothed and the effect of the noise is significantly reduced.

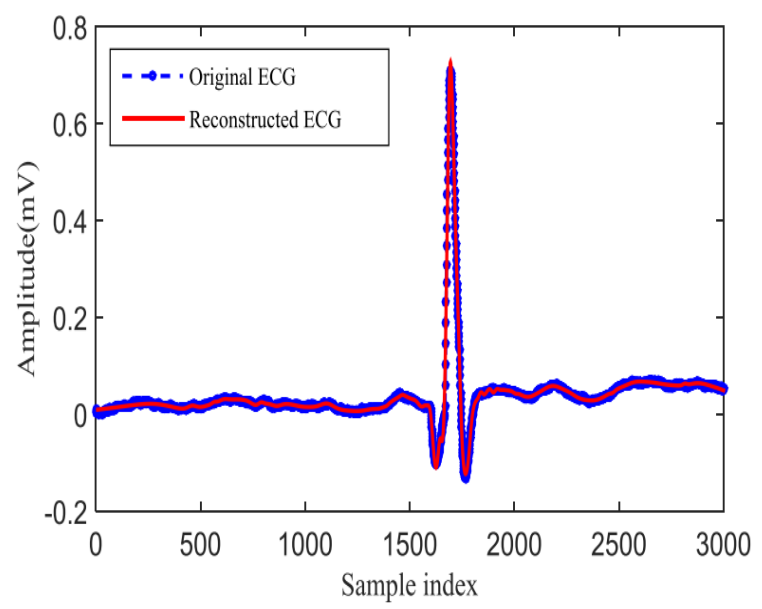

Figure (2): The original and reconstructed signals

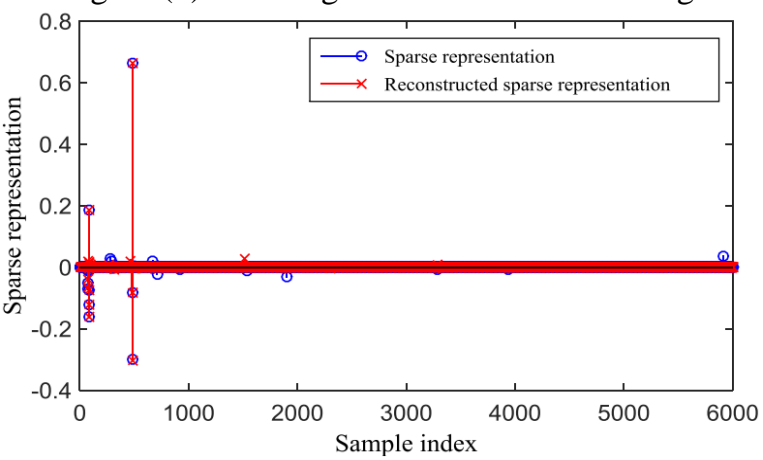

Figure (3): The signal sparse representation and its reconstructed form

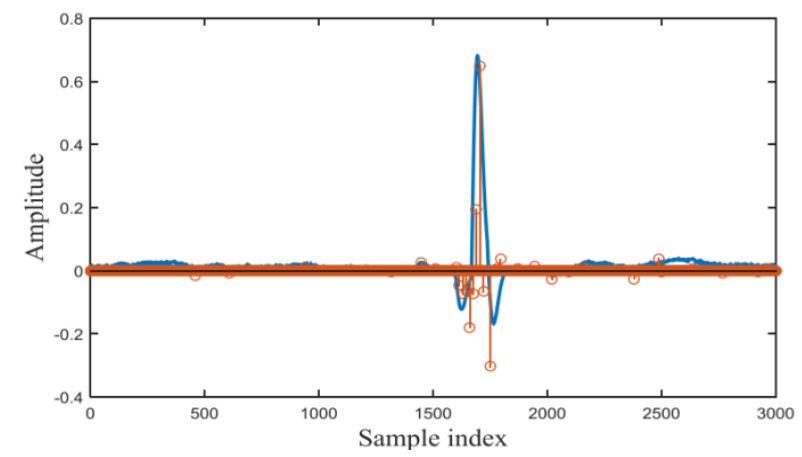

Figure (4): The original signal and its sparse representation

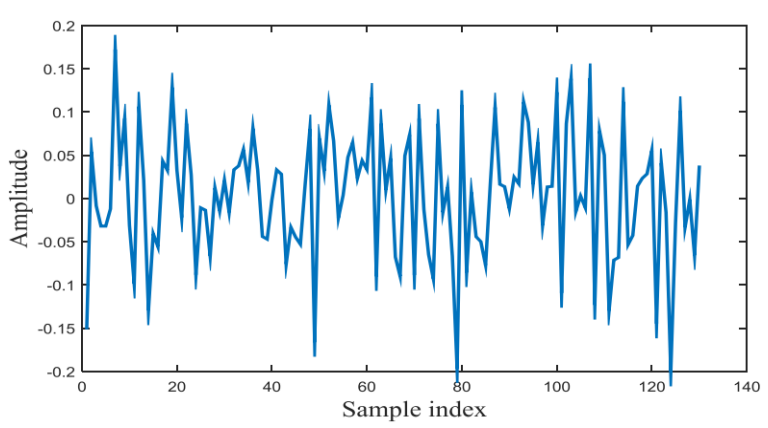

Figure (5): The compressed signal representation

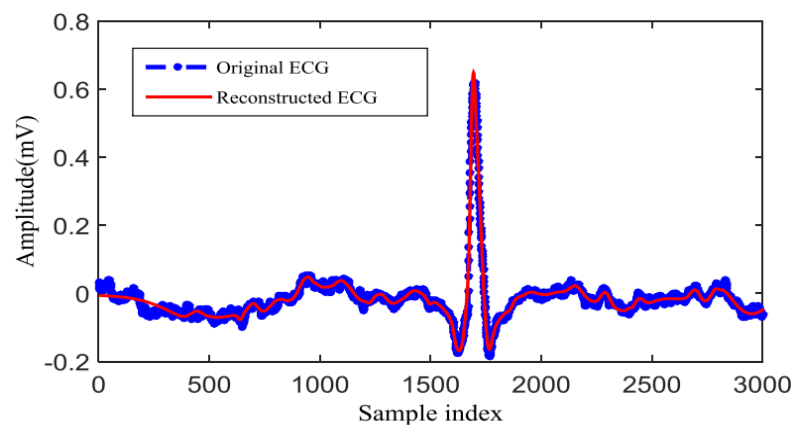

Figure (6): The original signal and its reconstruction with an increase in noise

Figure (7) shows the mean reconstruction error for the artificial ECG signal and the reconstructed signal with 100 iterations of the Monte Carlo test, which is determined via equation (10).

MeanError $=\frac{1}{100}\left(\sum_{\mathrm{i}=1}^{100} \sum_{\mathrm{j}=1}^{100}\left(\mathrm{R}_{\mathrm{i}}(\mathrm{j})-\mathrm{x}_{\mathrm{i}}(\mathrm{j})\right)\right)$

Where $\mathbf{x}$ and $\hat{\mathrm{X}}$ are the original and the reconstructed signal, respectively. 


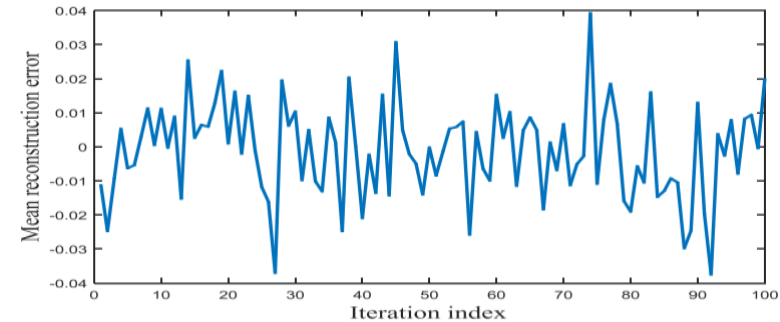

Figure (7): The mean reconstruction error for the original signal and the reconstructed signal

Afterward, the compression ratio $\mathrm{CR}=\mathrm{N} / \mathrm{m}$ is introduced in order to evaluate the performance of the proposed algorithm and its compression potential. In $\mathrm{CR}, \mathrm{m}$ is the length of the compressed signal and $\mathrm{N}$ is the length of the original signal. So, CR varies from 1 to $\infty(1 \leq \mathrm{CR}<\infty)$. The relationship between $\mathrm{CR}$ and the reconstruction error for three different $\mathrm{N}$ values is calculated through the Monte Carlo simulation.

Error $=\sum_{j=1}^{N}(\mathbb{x}(j)-x(j))^{2}$

In Figure (8), the reconstruction error versus different values of $\mathrm{CR}$ is depicted for different numbers of $\mathrm{N}$. The ranges in the three diagrams are different due to the difference in values of $\mathrm{N}$.

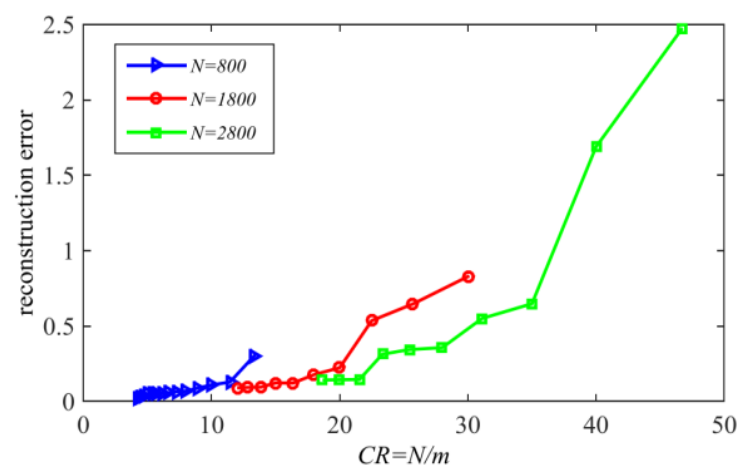

Figure (8): The compression ratio and the related reconstruction error

\subsection{Compression of the Real ECG Signal}

In this subsection, the proposed algorithm is applied to real multi-lead ECG signals and the results are compared with the algorithm proposed in [11], where the wavelet basis matrix is used. The simulation results show the considerable superiority of the proposed algorithm and the Gaussian basis matrix to the discrete wavelet basis. Fifteen real ECG signals at the sampling frequency of $360 \mathrm{~Hz}$ are obtained from Physionet database (Physiont ATM) [41]. The ECG signals belong to a 44-year-old woman, who does not smoke. Since the real ECG signals are noisy, some of the generated sparse coefficients are extra and belong to the noise of the signal. Hence, the additional coefficients are omitted using a thresholding method such that

$$
\begin{cases}1 & \left|\mathrm{c}_{\mathrm{i}}\right|>T \\ 0 & \left|\mathrm{c}_{\mathrm{i}}\right|<T\end{cases}
$$

If the generated sparse coefficient is larger than the threshold $\mathrm{T}$, the coefficient is considered significant. If it is smaller than the threshold $\mathrm{T}$, it is considered insignificant and equals zero. Using this thresholding method, the generated sparse coefficients decrease from 55 to 30. Figure (9) shows a period of the second lead of the multi-lead ECG signal database along with its sparse representation. In this figure, the sparse representation scale is modified to comply with the original signal. The original sparse representation, which is presented in Figure (10), shows on a dictionary with a size of $800 \times 6000$. Moreover, Figure (11) shows the compressed signal representation $y \in \mathcal{R}^{130 \times 1}$.

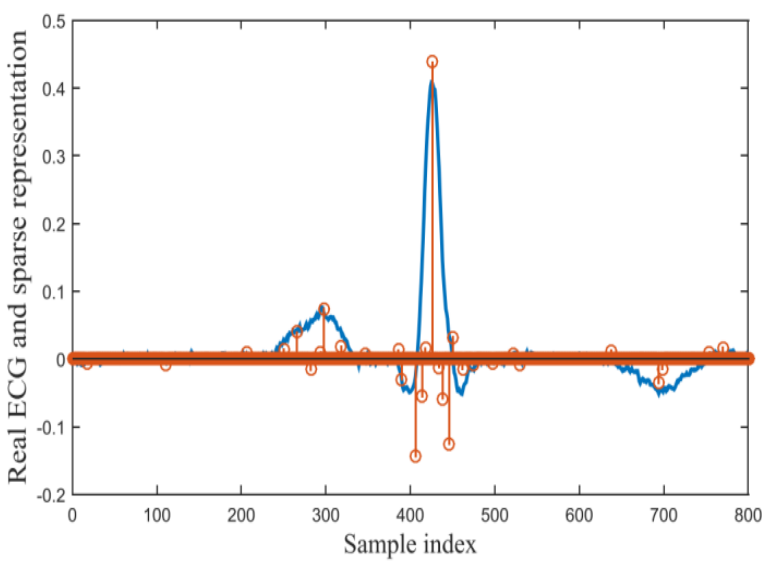

Figure (9): The real ECG signal and its sparse representation from the Physionet database.

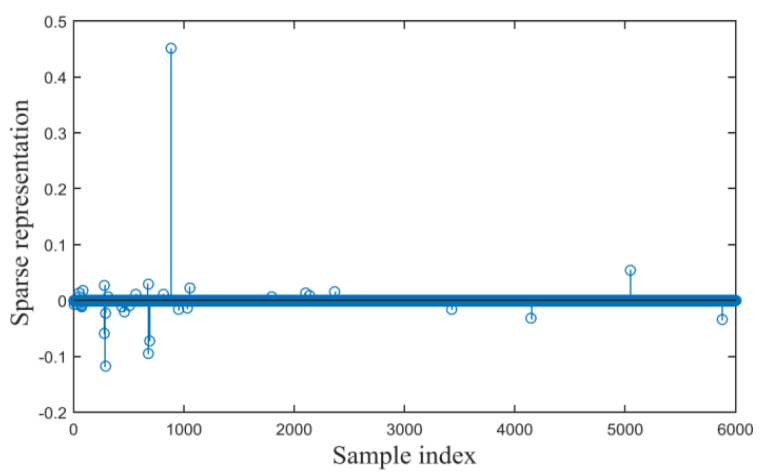

Figure (10): The sparse representation of the 2nd lead of the real multi-lead ECG signal from the Physionet database. 


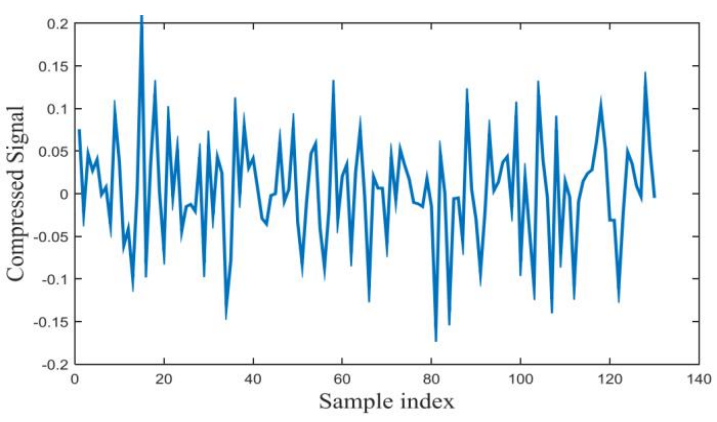

Figure (11): The compressed signal using compressive sensing.

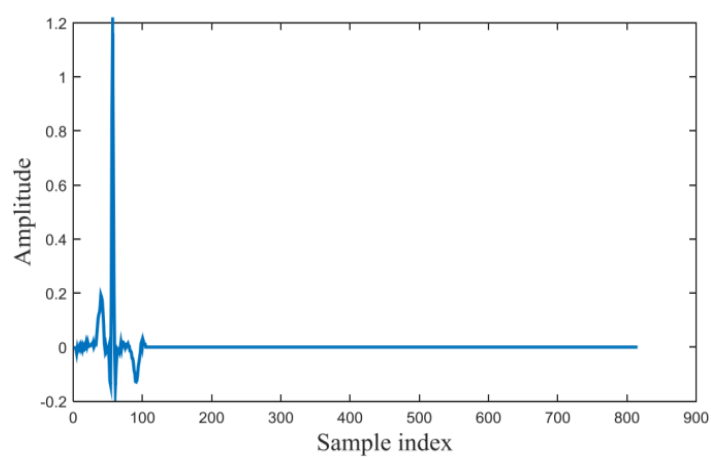

Figure (12): The sparse representation of the 2nd lead of the real multi-lead ECG signal from the Physionet database.

Besides, the ECG signal is turned into its sparse form using the wavelet basis matrix similar to [8] and the compressed sensing is applied to the signal. To this end, the Daubechies mother wavelet (db3) is used and the wavelet transform is implemented in three steps. As seen in Figure (12), the signal sparsity in this state equals 104. It is shown that the minor coefficients are equal to zero in the sparse representation.

Then the proposed algorithm is applied separately for each lead and then the signals are reconstructed. The original and reconstructed signals representations for each 15 lead ECG signal are depicted in Figure (13). The compliance of each single reconstructed signal with the original signals despite the high compression ratio demonstrates the accuracy of the proposed algorithm.

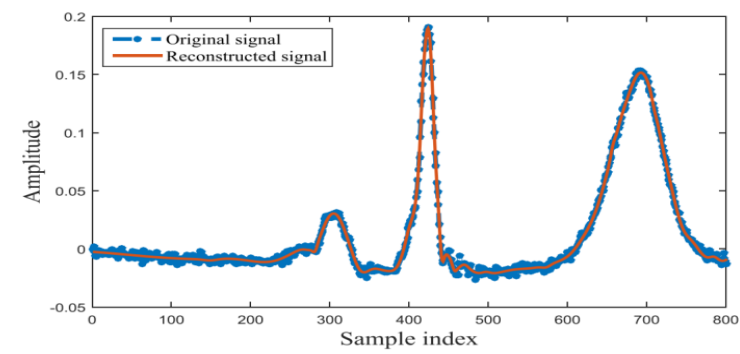

A) the first lead of multi-lead ECG database

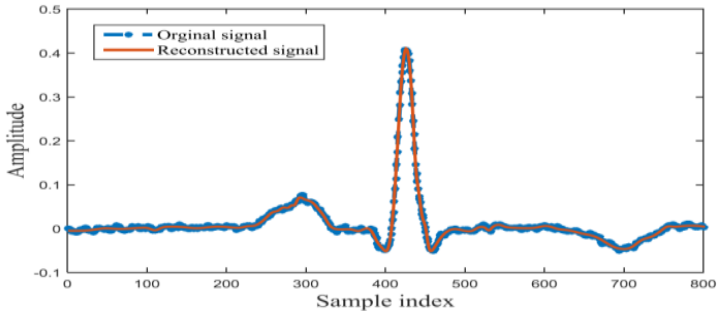

B) the second lead of multi-lead ECG database

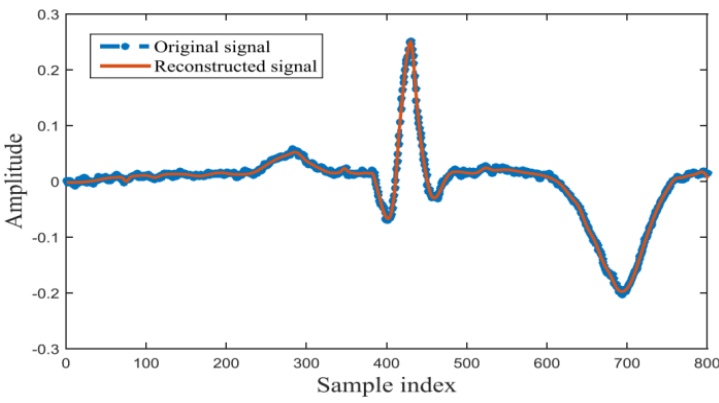

C) the third lead of multi-lead ECG database

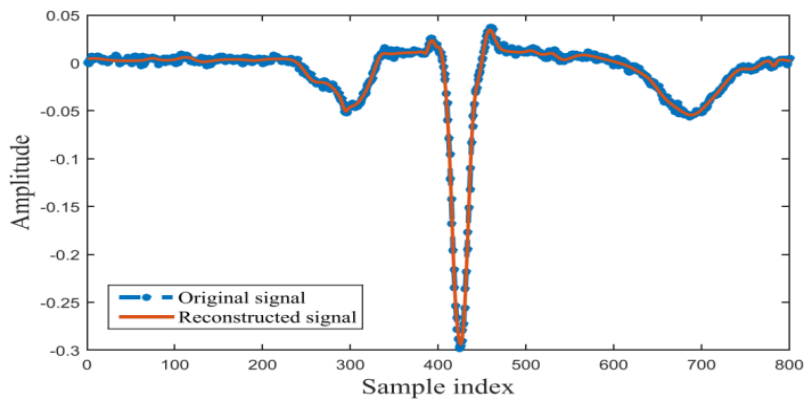

D) the fourth lead of multi-lead ECG database

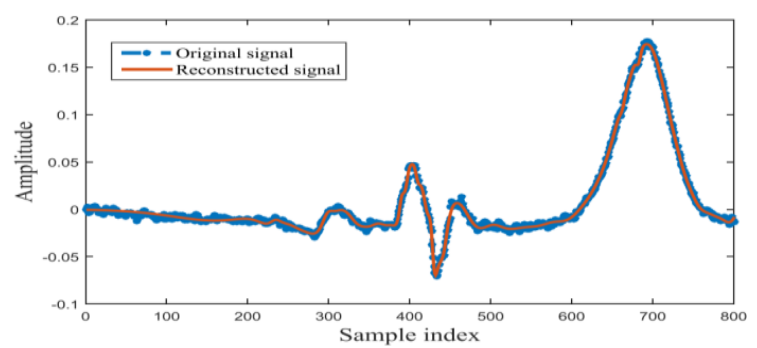

E) the fifth lead of multi-lead ECG database

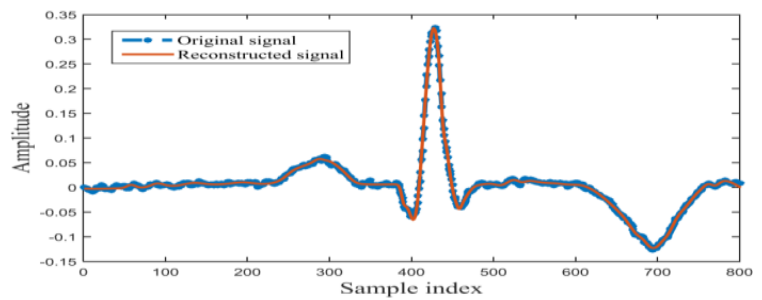

F) the sixth lead of multi-lead ECG database 


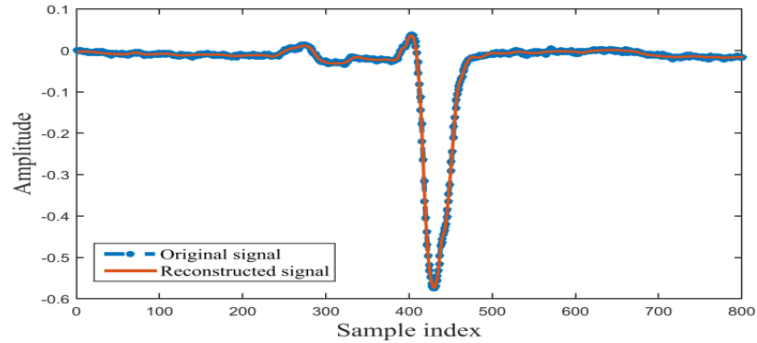

G) the seventh lead of multi-lead ECG database

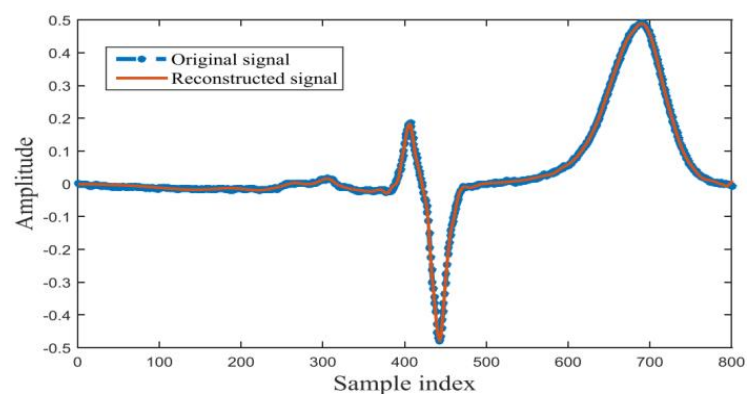

H) the eighth lead of multi-lead ECG database

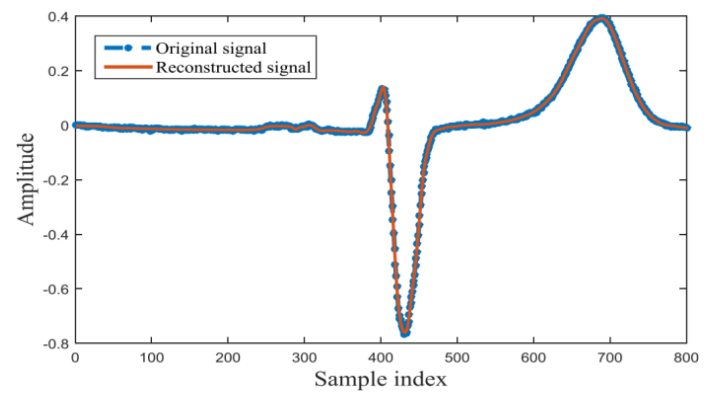

I) the ninth lead of multi-lead ECG database

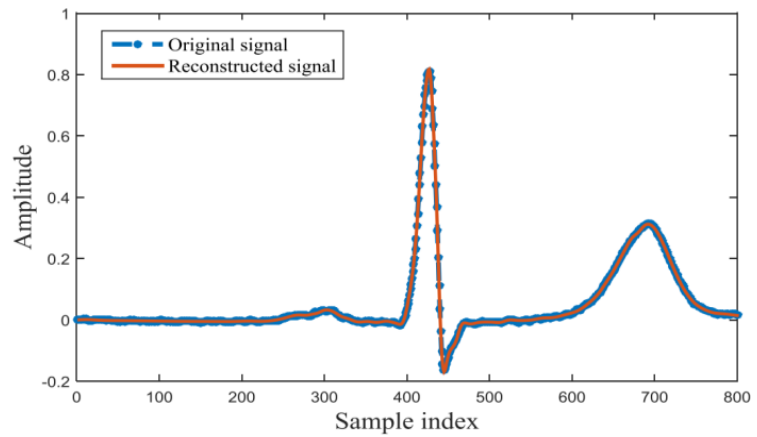

J) the tenth lead of multi-lead ECG database

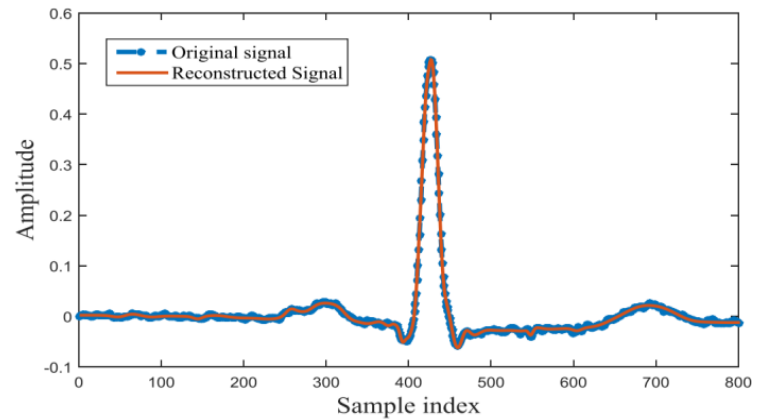

K) the eleventh lead of multi-lead ECG database

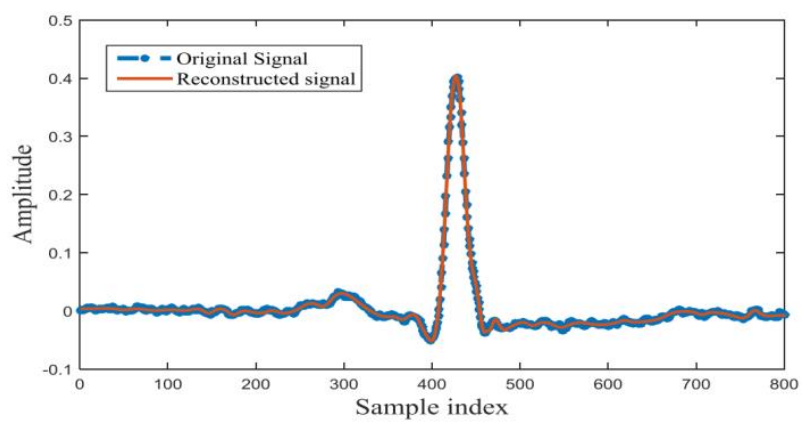

L) the twelve lead of multi-lead ECG database

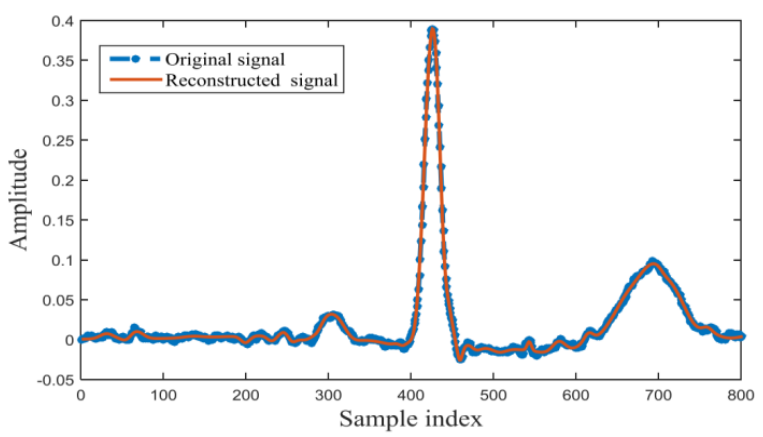

M) the thirteenth lead of multi-lead ECG database

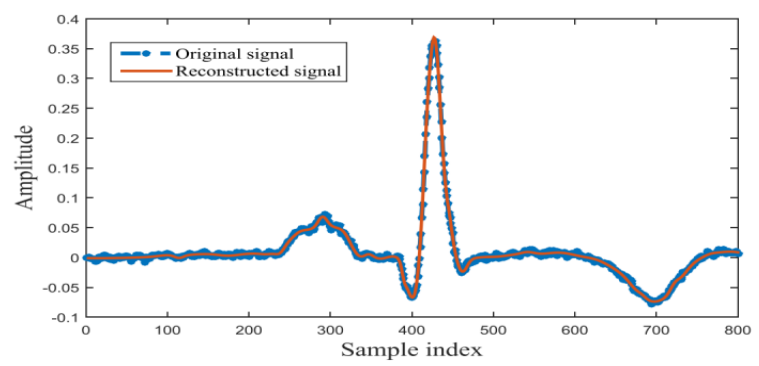

N) the fourteenth lead of multi-lead ECG database 


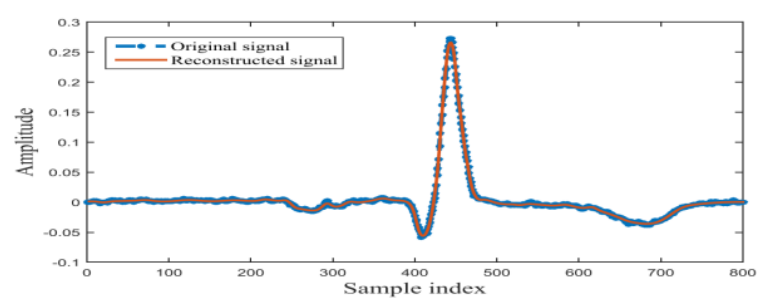

O) the fifteenth lead of multi-lead ECG database

Figure (13): The original and reconstructed signal by the proposed algorithm (A to $\mathrm{O}$ figures)

The reconstruction error of the multi-lead ECG signals is evaluated for the proposed algorithm and the algorithm in [11] in terms of different CR values. Thereafter, the errors of all ECG signals from the Physionet database are summed up and the average value is depicted in Figure (14). The superiority of the proposed algorithm and the Gaussian basis matrix to the wavelet basis matrix is clearly evident. The simulation results for six different people from the Physionet database are listed in Table (2). The first column in this table contains the reconstruction error for the proposed algorithm using the Gaussian basis functions, and the second column shows the reconstruction error for the wavelet basis functions. The improvement in the average quality is shown in the last row of this table, which confirms the better performance of the compression algorithm in the proposed method.

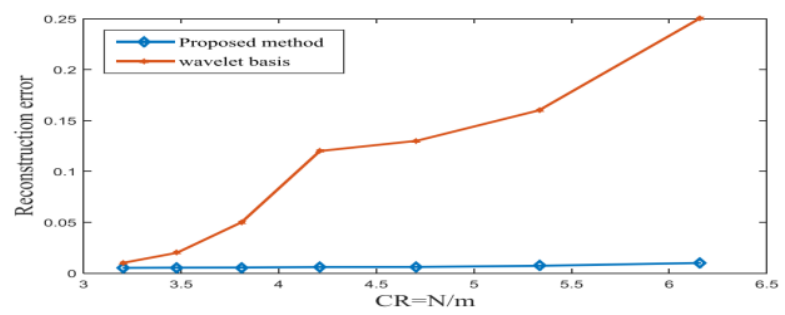

Figure (14): The mean reconstruction error of the multilead ECG signals for the proposed algorithm and the algorithm in [11] in terms of several different compression ratios.

Table (2): The reconstruction error for the proposed algorithm using Gaussian and wavelet kernels

\begin{tabular}{l|c|c}
\hline Subject no. & $\begin{array}{c}\text { OMP } \\
\text { (Gaussian } \\
\text { kernel) }\end{array}$ & $\begin{array}{c}\text { OMP } \\
\text { (Wavelet } \\
\text { kernel) }\end{array}$ \\
\hline S01901rem & $\mathbf{0 . 0 3 2 1}$ & 0.2327 \\
S0195lrem & $\mathbf{0 . 0 0 5 5}$ & 0.177 \\
S02421rem & $\mathbf{0 . 0 0 7 7}$ & 0.2702 \\
S0327lrem & $\mathbf{0 . 0 1 2 3}$ & 1.341 \\
S0031lrem & $\mathbf{0 . 0 0 3 6}$ & 0.33 \\
S0138lrem & $\mathbf{0 . 0 4 4}$ & 0.563 \\
Average \\
improvement \\
(\%)
\end{tabular}

\section{CONCLUSIONS}

In this paper, a new method is proposed for modelling the ECG signals and compressing these signals based on the compressed sensing theory. First, the sparse representation of the signals is required to implement the method. The sparse representation of each single signal is obtained using a basis matrix with Gaussian kernels. Thereafter, the compressed sensing is used for signal compression with very fewer samples than the original signal. To reconstruct the multi-lead ECG signals, the greedy optimization method is improved to reconstructed signals with high accuracy and low recovery error. This method was used for artificial and natural ECG signals. Simulation resultsverify that our proposed algorithm achieves higher reconstruction accuracy with a smaller number of required transmissions, reconstruction error as compared to the method with a wavelet basis.

\section{REFERENCES}

[1] D. Craven, B. McGinley, L. Kilmartin, M. Glavin, E. Jones, 'Compressed Sensing for Bioelectric Signals: A Review,' IEEE Journal of Biomedical and Health Informatics, 2015, 19, (2), pp. 529-540.

[2] Donoho, D.L. Compressed sensing. IEEE Transaction on Information Theory, 2006, vol. 52, no. 4, p. 1289-1306.

[3] H. Mamaghanian, N. Khaled, D. Atienza, P. Vandergheynst, 'Compressed sensing for real-time energy-efficient ECG compression on wireless body sensor nodes', IEEE Transactions on Biomedical Engineering, 2011, 58, (9), pp. 24562466.

[4] A. M. R. Dixon, E. G. Allstot, D. Gangopadhyay, D. J. Allstot, 'Compressed sensing system considerations for ECG and EMG wireless biosensors', IEEE Transactions on Biomedical Circuits Systems, 2012, 6, (2), pp. 156-166.

[5] Frankel, D.S., Model driven architecture: applying MDA to enterprise computing. 2003. Google Scholar Google Scholar Digital Library Digital Library.

[6] Cox, J. R., Nolle, F. M., Fozzard, H. A., \& Oliver, G. C. (1968). AZTEC, a preprocessing program for real-time ECG rhythm analysis. IEEE Transactions on Biomedical Engineering, (2), 128129.

[7] Abenstein, J. P., \& Tompkins, W. J. (1982). A new data-reduction algorithm for real-time ECG analysis. IEEE Transactions on Biomedical Engineering, (1), 43-48.

[8] Ishijima, M., Shin, S. B., Hostetter, G. H., \& Sklansky, J. (1983). Scan-along polygonal approximation for data compression of 
electrocardiograms. IEEE Transactions on Biomedical Engineering, (11), 723-729.

[9] Al-Shrouf, A., Abo-Zahhad, M., \& Ahmed, S. M. (2003). A novel compression algorithm for electrocardiogram signals based on the linear prediction of the wavelet coefficients. Digital Signal Processing, 13(4), 604-622.

[10] Al-Nashash, H. A. M. (1995). A dynamic Fourier series for the compression of ECG using FFT and adaptive coefficient estimation. Medical engineering \& physics, 17(3), 197-203.

[11] Raeiatibanadkooki, M., Quchani, S. R., KhalilZade, M., \& Bahaadinbeigy, K. (2016). Compression and encryption of ECG signal using wavelet and chaotically Huffman code in telemedicine application. Journal of medical systems, 40(3), 73.

[12] Lu, Z., Kim, D. Y., \& Pearlman, W. A. (2000). Wavelet compression of ECG signals by the set partitioning in hierarchical trees algorithm. IEEE transactions on Biomedical Engineering, 47(7), 849-856.

[13] Parkale, Y. V., \& Nalbalwar, S. L. (2017). Application of compressed sensing (CS) for ECG signal compression: A review. In Proceedings of the International Conference on Data Engineering and Communication Technology (pp. 53-65). Springer, Singapore.

[14] Satija, U., Ramkumar, B., \& Manikandan, M. S. (2018). An automated ECG signal quality assessment method for unsupervised diagnostic systems. Biocybernetics and Biomedical Engineering, 38(1), 54-70.

[15] Kumar, S., Deka, B., \& Datta, S. (2019, October). Weighted Block Compressed Sensing for Multichannel Fetal ECG Reconstruction. In TENCON 2019-2019 IEEE Region 10 Conference (TENCON) (pp. 2324-2328). IEEE.

[16] Mamaghanian, H., Ansaloni, G., Atienza, D., \& Vandergheynst, P. (2014, May). Power-efficient joint compressed sensing of multi-lead ECG signals. In 2014 IEEE International Conference on Acoustics, Speech and Signal Processing (ICASSP) (pp. 4409-4412).IEEE.

[17] Polania, L. F., Carrillo, R. E., Blanco-Velasco, M., \& Barner, K. E. (2014). Exploiting prior knowledge in compressed sensing wireless ECG systems. IEEE journal of Biomedical and Health Informatics, 19(2), 508-519.

[18] Yin, H., Li, J., Chai, Y., \& Yang, S. X. (2014). A survey on distributed compressed sensing: theory and applications. Frontiers of Computer Science, 8(6), 893-904.

[19] Yu, S., Liu, B., Qiao, W., Zhang, C., Chen, C. W., \& Cai, J. (2013, October). Jsm-2 based ecg compression with statistical support prediction. In 2013 IEEE 15th International Conference on e-
Health Networking, Applications and Services (Healthcom 2013) (pp. 218-222). IEEE.

[20] Mamaghanian, H., Khaled, N., Atienza, D. Vandergheynst, P. (2011). 'Compressed sensing for real-time energy efficient ECG compression on wireless body sensor nodes,' IEEE Transactions On Biomedical Engineering, 58, (9), 2456-2466.

[21] Abo-Zahhad, M. M., Hussein, A. I., Mohamed, A. M. (2015). 'Compression of ECG signal based on compressive sensing and the extraction of significant features,' International Journal of Communications, Network and System Sciences, 8, (4), 97-117.

[22] Zhang, J., Gu, Z., Yu, Z., Li, Y. (2015). 'Energyefficient ECG compression on wireless biosensors via minimal coherence sensing and weighted $l_{1}$ minimization reconstruction,' IEEE Journal of Biomedical and Health Informatics, 19, (2), 520528.

[23] Bortolotti, D., Mangia, M., Bartolini, A., Rovatti, R., Setti, G., Benini, L. (2018). 'Energy-aware biosignal compressed sensing reconstruction on the WBSN-gateway,' IEEE Transactions on Emerging Topics in Computing, 6, (3), 370-381.

[24] Dey, N., Ashour, A. S., Shi, F., Fong, S. J., Sherratt, R. S. (2017). 'Developing residential wireless sensor networks for ECG healthcare monitoring,' IEEE Transactions on Consumer Electronics, 63, (8), 442-449.

[25] Zonoobi, D., Kassim, A. A. (2014). 'On ECG reconstruction using weighted-compressive sensing,' Healthcare Technology Letters, 1, (2), 68-73.

[26] Polanía, L. F., Carrillo, R. E., Blanco-Velasco, M., Barner, K. E. (2015), 'Exploiting prior knowledge in compressed sensing wireless ECG systems,' IEEE Journal of Biomedical and Health Informatics, 19, (2), 508-519.

[27] Craven, D., McGinley, B., Kilmartin, L., Glavin, M., Jones, E. (2016). 'Energy-efficient compressed sensing for ambulatory ECG monitoring.' Computers in Biology and Medicines, 71, (6), 1-3.

[28] Craven, D., McGinley, B., Kilmartin, L., Glavin, M., Jones, E. (2015). 'Impact of compressed sensing on clinically relevant metrics for ambulatory ECG monitoring,' Electronics Letters, 51, 323-325.

[29] Polanía, L. F., M., Barner, K. E. (2013). 'Multiscale dictionary learning for compressive sensing ECG,' Proceedings of the IEEE Digital Signal Processing and Signal Processing Education Meeting (DSP/SPE), 36- 41.

[30] Kumar, R. Kumar, A., Singh, G. K., Lee, H. N. (2017). 'Efficient compression technique based on temporal modelling of ECG signal using principle component analysis,' IET Science Measurement and Technology, 11, (3), 346-353. 
[31] Cheng, Y., Ye, Y., Hou, M., He, W., Li, Y., Deng, $X$. (2018). 'A fast and robust non-sparse signal recovery algorithm for wearable ECG telemonitoring using ADMM-based block sparse Bayesian learning,' Sensors, 18, (7), 2021-2034.

[32] Jahanshahi, J. A., Danyali, H., Helfroush, M. S. (2020), 'Compressive Sensing based the MultiChannel ECG reconstruction in Wireless Body Sensor Networks,' Biomedical Signal Processing and Control, 61, (10), 2021-2034.

[33] McSharry, P. E., Clifford, G. D., Tarassenko, L., \& Smith, L. A. (2003). A Dynamic Model for Generating Synthetic Electrocardiogram Signals. IEEE Trans. Biomed. Eng.

[34] Söderström, T. (2002). Discrete-time stochastic systems: estimation and control. Springer Science \& Business Media.

[35] Candes, E. J., \& Tao, T. (2006). Near-optimal signal recovery from random projections: Universal encoding strategies?. IEEE transactions on information theory, 52(12), 5406-5425.

[36] Tropp, J. A., \& Gilbert, A. C. (2007). Signal recovery from random measurements via orthogonal matching pursuit. IEEE Transactions on information theory, 53(12), 4655-4666.

[37] Needell, D., \& Tropp, J. A. (2009). CoSaMP: Iterative signal recovery from incomplete and inaccurate samples. Applied and computational harmonic analysis, 26(3), 301-321.

[38] Donoho, D. L., Tsaig, Y., Drori, I., \& Starck, J. L. (2012). Sparse solution of underdetermined systems of linear equations by stagewise orthogonal matching pursuit. IEEE transactions on Information Theory, 58(2), 1094-1121.

[39] Eldar, Y. C., \& Kutyniok, G. (Eds.). (2012). Compressed sensing: theory and applications. Cambridge university press.

[40] Behar, J., Andreotti, F., Zaunseder, S., Oster, J., \& Clifford, G. D. (2016). A practical guide to noninvasive foetal electrocardiogram extraction and analysis. Physiological measurement, 37(5), R1.

[41] The MIT-BIH PTB diagnosis database; http://www.physionet.org/physiobank/database/ptb $\mathrm{db} /$. 